\title{
SOME LOWER BOUNDS FOR THE PERRON ROOT OF A NONNEGATIVE MATRIX
}

\author{
SHU-QIAN SHEN AND GUANG-BIN WANG
}

\begin{abstract}
In this paper, we present some lower bounds for the Perron root of a symmetric nonnegative matrix, which are then applied to give the lower bounds of the Perron root of a general nonnegative matrix. These bounds improve the corresponding ones in [3] and [5]. Numerical examples are supplemented to illustrate the effectiveness of the presented bounds.
\end{abstract}

Mathematics subject classification (2010): 15A18, 15A57, 65F15.

Keywords and phrases: Nonnegative matrices, Perron roots, lower bounds.

\section{REFERENCES}

[1] A. Berman and R. J. Plemmons, Nonnegative Matrices in the Mathematical Sciences, Academic Press, New York, 1979.

[2] A. Brauer AND I. C. Gentry, Bounds for the greatest characteristic root of an irreducible nonnegative matrix, Linear Algebra Appl. 8, 2 (1974), 105-107.

[3] A. BRAUER AND I. C. Gentry, Bounds for the greatest characteristic root of an irreducible nonnegative matrix II, Linear Algebra Appl. 13, 1-2 (1976), 109-114.

[4] E. DeUtSCH AND H. WielandT, Nested bounds for the Perron root of a nonnegative matrix, Linear Algebra Appl. 52/53, 1 (1983), 235-251.

[5] L. Yu. Kolotilina, Lower bounds for the Perron root of a nonnegative matrix, Linear Algebra Appl. 180, 1 (1993), 133-151.

[6] L. YU. Kolotilina, Bounds and inequalities for the Perron root of a nonnegative matrix, J. Math. Sci. 121, 4 (2004), 2481-2507.

[7] L. Z. Lu, Perron complement and Perron root, Linear Algebra Appl. 341, 1-3 (2002), 239-248.

[8] L. Z. LU AND M. K. NG, Localization of Perron roots, Linear Algebra Appl. 392, 1 (2004), 103-117.

[9] J. K. Merikos Ki AND A. VirTANEn, The best possible lower bound for the Perron root using traces, Linear Algebra Appl. 388, 1 (2004), 301-313.

[10] H. Minc, Nonnegative Matrices, John Wiley and Sons, New York, 1988.

[11] A. J. SCHWENK, Tight bounds on the spectral radius of asymmetric nonnegative matrices, Linear Algebra Appl. 75, 1 (1986), 257-265.

[12] Y. Z. SonG, Lower bounds for the Perron root of a nonnegative matrix, Linear Algebra Appl. 169, 1 (1992), 269-278.

[13] G. W. Stewart And J. G. Sun, Matrix Perturbation Theory, Academic Press, London, 1990.

[14] T. SzULC, A lower bound for the Perron root of a nonnegative matrix, Linear Algebra Appl. 101, 1 (1988), 181-186.

[15] T. SzULC, A lower bound for the Perron root of a nonnegative matrix. II, Linear Algebra Appl. 112, 1 (1989), 19-27. 